\title{
The Attractor Mechanism in Five Dimensions
}

\author{
Finn Larsen \\ Michigan Center for Theoretical Physics, Department of Physics \\ University of Michigan, Ann Arbor, MI-48109, USA. \\ larsenf@umich.edu
}

\begin{abstract}
We give a pedagogical introduction to the attractor mechanism. We begin by developing the formalism for the simplest example of spherically symmetric black holes in five dimensions which preserve supersymmetry. We then discuss the refinements needed when spherical symmetry is relaxed. This is motivated by rotating black holes and, especially, black rings. An introduction to nonBPS attractors is included, as is a discussion of thermodynamic interpretations of the attractor mechanism. ${ }^{1}$
\end{abstract}

\section{Introduction}

These lectures are intended as a pedagogical introduction to the attractor mechanism. With this mission in mind we will seek to be explicit and, to the extent possible, introduce the various ingredients using rather elementary concepts. While this will come at some loss in mathematical sophistication, it should be helpful to students who are not already familiar with the attractor mechanism and, for the experts, it may serve to increase transparency.

A simple and instructive setting for studying the attractor mechanism is M-theory compactified to five dimensions on a Calabi-Yau three-fold. The resulting low energy theory has $N=2$ supersymmetry and it is based on real special geometry. We will focus on this setting because of the pedagogical mission of the lectures: real special geometry is a bit simpler than complex special geometry, underlying $N=2$ theories in four dimensions.

\footnotetext{
${ }^{1}$ Lectures presented at the Winter School on the Attractor Mechanism (Frascati, March 20-24, 2006).
} 
The simplest example where the attractor mechanism applies is that of a regular, spherically symmetric black hole that preserves supersymmetry. In the first lecture we develop the attractor mechanism in this context, and then verify the results by considering the explicit black hole geometry.

In the second lecture we generalize the attractor mechanism to situations that preserve supersymmetry, but not necessarily spherical symmetry. Some representative examples are rotating black holes, multi-center black holes, black strings, and black rings. Each of these examples introduce new features that have qualitative significance for the implementation of the attractor mechanism. The approach will follow the paper [1] rather closely, with the difference that here we include many more examples and other pedagogical material that should be helpful when learning the subject.

In the third lecture we consider an alternative approach to the attractor mechanism which amount to seeing the attractor behavior as a result of an extremization procedure, rather than a supersymmetric flow. One setting that motivates this view is applications to black holes that are extremal, but not supersymmetric. Extremization principles makes it clear that the attractor mechanism applies to such black holes as well.

Another reason for the interest in extremization principles is more philosophical: we would like to understand what the attractor mechanism means in terms of physical principles. There does not yet seem to be a satisfactory formulation that encompasses all the different examples, but there are many interesting hints.

The literature on the attractor mechanism is by now enormous. As general references let us mention from the outset the original works 2 establishing the attractor mechanism. It is also worth highlighting the review [3] which considers the subject using more mathematical sophistication than we do here. In view of the extensive literature on the subject we will not be comprehensive when referencing. Instead we generally provide just a few references that may serve as entry points to the literature.

\section{The Basics of the Attractor Mechanism}

In this section we first introduce a few concepts from the geometry of CalabiYau spaces and real special geometry. We then review the compactification of eleven-dimensional supergravity on a Calabi-Yau space and the resulting $N=$ 2 supergravity Lagrangian in five dimensions. This sets up a discussion of the attractor mechanism for spherically symmetric black holes in five dimensions. We conclude the lecture by giving explicit formulae in the case of toroidal compactification.

\subsection{Geometrical Preliminaries}

On a complex manifold with hermitian metric $g_{\mu \bar{\nu}}$ it is useful to introduce the Kähler two-form $J$ through 


$$
J=i g_{\mu \bar{\nu}} d z^{\mu} \wedge d z^{\bar{\nu}} .
$$

Kähler manifolds are complex manifolds with hermitian metric such that the corresponding Kähler form is closed, $d J=0$. The linear space spanned by all closed $(1,1)$ forms (modulo exact forms) is an important structure that is known as the Dolbault cohomology and given the symbol $H_{\bar{a}}^{1,1}$. If we denote by $J_{I}$ a basis of this cohomology we can expand the closed Kähler form as

$$
J=X^{I} J_{I} \quad ; I=1, \ldots, h_{11} .
$$

This expansion is a statement in the sense of cohomology so it should be understood modulo exact forms.

Introducing the basis $(1,1)$-cycles $\Omega^{I}$ we can write the expression

$$
X^{I}=\int_{\Omega^{I}} J \quad ; I=1, \ldots, h_{11},
$$

for the real expansion coefficients $X^{I}$ in (2). We see that they can be interpreted geometrically as the volumes of $(1,1)$-cycles within the manifold. The $X^{I}$ are known as Kähler moduli. In the context of compactification the Kähler moduli become functions on spacetime and so the $X^{I}$ will be interpreted as scalar fields.

One of several ways to define a Calabi-Yau space is that it is a Kähler manifold that permits a globally defined holomorphic three-form. One consequence of this property is that Calabi-Yau spaces do not have any $(0,2)$ and $(2,0)$ forms. For this reason the $(1,1)$ cycles $\Omega^{I}$ are in fact the only two-cycles on the manifold.

The two-cycles $\Omega^{I}$ give rise to a dual basis of four-cycles $\Omega_{I}, I=1, \ldots, h_{11}$, constructed such that their intersection numbers with the two-cycles are canonical $\left(\Omega^{I}, \Omega_{J}\right)=\delta_{J}^{I}$. The volumes of the four-cycles are measured by the Kähler form as

$$
X_{I}=\frac{1}{2} \int_{\Omega_{I}} J \wedge J .
$$

The integral can be evaluated by noting that the two-form $J_{I}$ covers the space transverse to the 4-cycle $\Omega_{I}$. Therefore

$$
X_{I}=\frac{1}{2} \int_{C Y} J \wedge J \wedge J_{I}=\frac{1}{2} C_{I J K} X^{J} X^{K},
$$

where the integrals

$$
C_{I J K}=\int_{C Y} J_{I} \wedge J_{J} \wedge J_{K},
$$

are known as intersection numbers because they count the points where the four-cycles $\Omega_{I}, \Omega_{J}$ and $\Omega_{K}$ all intersect. 


\subsection{The Effective Theory in Five Dimensions}

We next review the compactification of M-theory on a Calabi-Yau manifold 4. The resulting theory in five dimensions can be approximated at large distances by $N=2$ supergravity. In addition to the $N=2$ supergravity multiplet, the low energy theory will include matter organized into a number of $N=2$ vector multiplets and hypermultiplets. In discussions of the attractor mechanism the hyper-multiplets decouple and can be neglected. We therefore focus on the gravity multiplet and the vector multiplets.

The $N=2$ supergravity multiplet in five dimensions contains the metric, a vector field, and a gravitino (a total of $8+8$ physical bosons + fermions). Each $N=2$ vector multiplet in five dimensions contains a vector field, a scalar field, and a gaugino (a total of $4+4$ physical bosons+fermions). It is useful to focus on the vector fields. These fields all have their origin in the three-form in eleven dimensions which can be expanded as

$$
\mathcal{A}=A^{I} \wedge J_{I} \quad ; I=1, \ldots, h_{11} .
$$

The $J_{I}$ are the elements of the basis of $(1,1)$ forms introduced in (2). Among the $h_{11}$ gauge fields $A^{I}, I=1, \ldots, h_{11}$, the linear combination

$$
A^{\text {grav }}=X_{I} A^{I}
$$

is a component of the gravity multiplet. This linear combination is known as the graviphoton. The remaining $n_{V}=h_{11}-1$ vector fields are components of $N=2$ vector multiplets.

The scalar components of the vector multiplets are essentially the scalar fields $X^{I}$ introduced in (3). The only complication is that, since one of the vector fields does not belong to a vector multiplet, it must be that one of the scalars $X^{I}$ also does not belong to a vector multiplet. Indeed, it turns out that the overall volume of the Calabi-Yau space

$$
\mathcal{V}=\frac{1}{3 !} \int_{C Y} J \wedge J \wedge J=\frac{1}{3 !} C_{I J K} X^{I} X^{J} X^{K}
$$

is in a hyper-multiplet. As we have already mentioned, hyper-multiplets decouple and we do not need to keep track of them. Therefore (9) can be treated as a constraint that sets a particular combination of the $X^{I}$ 's to a constant. The truly independent scalars obtained by solving the constraint (9) are denoted $\phi^{i}, i=1, \ldots, n_{V}$. These are the scalars that belong to vector multiplets.

We now have all the ingredients needed to present the Lagrangean of the theory. The starting point is the bosonic part of eleven-dimensional supergravity

$$
S_{11}=\frac{1}{2 \kappa_{11}^{2}} \int\left[-R^{*} 1-\frac{1}{2} \mathcal{F} \wedge{ }^{*} \mathcal{F}-\frac{1}{3 !} \mathcal{F} \wedge \mathcal{F} \wedge \mathcal{A}\right]
$$

where the four-form field strength is $\mathcal{F}=d \mathcal{A}$. The coupling constant is related to Newton's constant as $\kappa_{D}^{2}=8 \pi G_{D}$. Reducing to five dimensions we find 
$S_{5}=\frac{1}{2 \kappa_{5}^{2}} \int\left[-R^{*} 1-G_{I J} d X^{I} \wedge^{*} d X^{J}-G_{I J} F^{I} \wedge^{*} F^{J}-\frac{1}{3 !} C_{I J K} F^{I} \wedge F^{J} \wedge A^{K}\right]$,

where $F^{I}=d A^{I}$ and $\kappa_{5}^{2}=\kappa_{11}^{2} / \mathcal{V}$. The hodge-star is now the five-dimensional one, although we have not introduced new notation to stress this fact.

The gauge kinetic term in (11) is governed by the metric

$$
G_{I J}=\frac{1}{2} \int_{C Y} J_{I} \wedge^{*} J_{J}
$$

It can be shown that

$$
G_{I J}=-\frac{1}{2} \partial_{I} \partial_{J}(\ln \mathcal{V})=-\frac{1}{2 \mathcal{V}}\left(C_{I J K} X^{K}-\frac{1}{\mathcal{V}} X_{I} X_{J}\right),
$$

where the notation $\partial_{I}=\frac{\partial}{\partial X^{T}}$. Combining (5) and (9) we have the relation

$$
X_{I} X^{I}=3 \mathcal{V}
$$

and so (13) gives

$$
G_{I J} X^{J}=\frac{1}{2 \mathcal{V}} X_{I}
$$

The metric $G_{I J}$ (and its inverse $G^{I J}$ ) thus lowers (and raises) the indices $I, J=1, \cdots, h_{11}$. It is sometimes useful to extend this action to the intersection numbers $C_{I J K}$ so that, e.g., the constraint (9) can be be reorganized as

$$
\mathcal{V}^{2}=\frac{1}{3 !} C^{I J K} X_{I} X_{J} X_{K}
$$

where all indices were either raised or lowered.

The effective action in five dimensions (11) was written in terms of the fields $X^{I}$ which include some redundancy because the constraint (9) should be imposed on them. An alternative form of the scalar term which employs only the unconstrained scalars $\phi^{i}$ is

$$
\mathcal{L}_{\text {scalar }}=-\frac{1}{2 \kappa_{5}^{2}} g_{i j} d \phi^{i} \wedge^{*} d \phi^{j},
$$

where the metric on moduli space is

$$
g_{i j}=G_{I J} \partial_{i} X^{I} \partial_{j} X^{J} .
$$

Here derivatives with respect to the unconstrained fields are

$$
\partial_{i} X^{I}=\frac{\partial X^{I}}{\partial \phi^{i}} .
$$

So far we have just discussed the bosonic part of the supergravity action. We will not need the explicit form of the terms that contain fermions. However, 
it is important that the full Lagrangean is invariant under the supersymmetry variations

$$
\begin{aligned}
\delta \psi_{\mu} & =\left[D_{\mu}(\omega)+\frac{i}{24} X_{I}\left(\Gamma_{\mu}^{\nu \rho}-4 \delta_{\mu}^{\nu} \Gamma^{\rho}\right) F_{\nu \rho}^{I}\right] \epsilon, \\
\delta \lambda_{i} & =-\frac{1}{2} G_{I J} \partial_{i} X^{I}\left[\frac{1}{2} \Gamma^{\mu \nu} F_{\mu \nu}^{J}+i \Gamma^{\mu} \partial_{\mu} X^{J}\right] \epsilon,
\end{aligned}
$$

of the gravitino $\psi_{\mu}$ and the gauginos $\lambda_{i}, i=1, \ldots, n_{V}$. Here $\epsilon$ denotes the infinitesimal supersymmetry parameter. $D_{\mu}(\omega)$ is the covariant derivative formed from the connection $\omega$ and acting on the spinor $\epsilon$. The usual $\Gamma$-matrices in five dimensions are denoted $\Gamma^{\mu}$; and their multi-index versions $\Gamma^{\mu \nu}$ and $\Gamma^{\mu \nu \rho}$ are fully anti-symmetrized products of those.

\subsection{A First Look at the Attractor Mechanism}

We have now introduced the ingredients we need for a first look at the attractor mechanism. For now we will consider the case of supersymmetric black holes. As the terminology indicates, such black holes preserve at least some of the supersymmetries. This means $\delta \psi_{\mu}=\delta \lambda_{i}=0$ for some components of the supersymmetry parameter $\epsilon$. A great deal can be learnt from these conditions by analyzing the explicit formulae (2021).

In order to make the conditions more explicit we will make some simplifying assumptions. First of all, we will consider only stationary solutions in these lectures. This means we assume that the configuration allows a time-like Killing vector. The corresponding coordinate will be denoted $t$. All the fields are independent of this coordinate. The supersymmetry parameter $\epsilon$ satisfies

$$
\Gamma^{\hat{t}} \epsilon=-i \epsilon,
$$

where hatted coordinates refer to a local orthonormal basis. In order to keep the discussion as simple and transparent as possible we will for now also assume radial symmetry. This last assumption is very strong and will be relaxed in the following lecture. At any rate, under these assumptions the gaugino variation (21) reads

$$
\delta \lambda_{i}=\frac{i}{2} G_{I J} \partial_{i} X^{I}\left(F_{m \hat{t}}^{J}-\partial_{m} X^{J}\right) \Gamma^{m} \epsilon=0,
$$

where $m$ is the spatial index. We exploited that, due to radial symmetry, only the electric components $F_{m \hat{t}}^{I}$ of the field strength can be nonvanishing. We next assume that the solution preserve $N=1$ supersymmetry so that (22) are the only projections imposed on the spinor $\epsilon$. Then $\Gamma^{m} \epsilon$ will be nonvanishing for all $m$ and the solutions to (23) must satisfy

$$
G_{I J} \partial_{i} X^{I}\left(F_{m \hat{t}}^{J}-\partial_{m} X^{J}\right)=0 .
$$


This is a linear equation that depends on the bosonic fields alone. It essentially states that the gradient of the scalar field (of type $J$ ) is identified with the electric field (of the same type). This identification is at the core of the attractor mechanism. Later we will take more carefully into account the presence of the overall projection operator $G_{I J} \partial_{i} X^{I}$ in (24). This operator takes into account that fact that no scalar field is a superpartner of the graviphoton. This restriction arises here because the index $i=1, \ldots, n_{V}$ enumerating the gauginos is one short of the vector field index $I=1, \ldots, n_{V}+1$.

The conditions (24) give rise to an important monotonicity property that controls the attractor flow. To see this, multiply by $\partial_{r} \phi^{i}$ and sum over $i$. After reorganization we find

$$
G_{I J} \partial_{r} X^{I} F_{r \hat{t}}^{J}=G_{I J} \partial_{r} X^{I} \partial_{r} X^{J} \geq 0 .
$$

The quantity on the right hand side of the equation is manifestly positively definite. In order to simplify the left hand of the equation we need to analyze Gauss' law for the flux. For spherically symmetric configurations the ChernSimons terms in the action (11) do not contribute so the Maxwell equation is just

$$
d\left(G_{I J}^{*} F^{J}\right)=0 .
$$

Using the explicit form of the metric for a radially symmetric extremal black hole in five dimensions

$$
d s^{2}=-f^{2} d t^{2}+f^{-1}\left(d r^{2}+r^{2} d \Omega_{3}^{2}\right),
$$

the component form of the corresponding Gauss' law reads

$$
\partial_{r}\left(G_{I J} r^{3} f^{-1} F_{r \hat{t}}^{J}\right)=0 .
$$

This can be integrated to give the explicit solution

$$
G_{I J} F_{r \hat{t}}^{J}=f \cdot \frac{1}{r^{3}} \cdot \text { const } \equiv f \cdot \frac{Q_{I}}{r^{3}},
$$

for the radial dependence of the electric field. Inserting this in (25) we find the flow equation

$$
\partial_{r}\left(X^{I} Q_{I}\right)=f^{-1} r^{3} G_{I J} \partial_{r} X^{I} \partial_{r} X^{J} \geq 0 .
$$

We can summarize this important result as the statement that the central charge

$$
Z_{e} \equiv X^{I} Q_{I}
$$

depends monotonically on the radial coordinate $r$. It starts as a maximum in the asymptotically flat space and decreases as the black hole is approached. This is the attractor flow.

In order to analyze the behavior of (30) close to the horizon it is useful to write it as 


$$
r \partial_{r} Z_{e}=f^{-2} r^{4} \epsilon \geq 0
$$

where the energy density in the scalar field is

$$
\epsilon=g^{r r} G_{I J} \partial_{r} X^{I} \partial_{r} X^{J}
$$

According to the line element (27) an event horizon at $r=0$ is characterized by the asymptotic behavior $f \sim r^{2}$. Therefore the measure factor $f^{-2} r^{4}$ is finite there. Importantly, when $f \sim r^{2}$ the proper distance to the horizon diverges as $\int_{0} d r / r$. Since the horizon area is finite this means the proper volume of the near horizon region diverges. This is a key property of extremal black holes. In the present discussion the important consequence is that the energy density of the scalars in the near horizon region must vanish, or else they would have infinite energy, and so deform the geometry uncontrollably. We conclude that the right hand side of (32) vanishes at the horizon, i.e. the inequality is saturated there. We therefore find the extremization condition

$$
r \partial_{r} Z_{e}=0, \quad(\text { at horizon })
$$

This is the spacetime form of the attractor formula.

There is another form of the attractor formula that is cast entirely in terms of the moduli space. To derive it, we begin again from (24), simplify using Gauss' law (29), and introduce the central charge (31). We can write the result as

$$
\partial_{i} Z_{e}=\sqrt{g_{\perp}} g_{i j} \partial_{n} \phi^{j}
$$

where $\sqrt{g_{\perp}}=f^{-3 / 2} r^{3}$ is the area element, $g_{i j}$ is the metric on moduli space introduced in (18), $\partial_{n}=\sqrt{g^{r r}} \partial_{r}$ is the proper normal derivative, and the $\phi^{j}$ are the unconstrained moduli. As discussed in the previous paragraph, the energy density (33) must vanish at the horizon for extremal black holes. This means the contribution from each of the unconstrained moduli must vanish by itself, and so the right hand side of (35) must vanish for all values of the index $i$. We can therefore write the attractor formula as an extremization principle over moduli space

$$
\partial_{i} Z_{e}=0, \quad \text { (at horizon) . }
$$

This form of the attractor formula determines the values $X_{\text {ext }}^{I}$ of the scalar fields at the horizon in terms of the charges $Q_{I}$.

We can solve (36) explicitly. In order to take the constraint (9) on the scalars properly into account it is useful to rewrite the extremization principle as

$$
D_{I} Z_{e}=0, \quad(\text { at horizon }),
$$

where the covariant derivative is defined as

$$
D_{I} Z_{e}=\left(\partial_{I}-\frac{1}{3}\left(\partial_{I} \ln \mathcal{V}\right)\right) Z_{e}=\left(\partial_{I}-\frac{1}{3 \mathcal{V}} X_{I}\right) Z_{e}=Q_{I}-\frac{1}{3 \mathcal{V}} X_{I} Z_{e}
$$


We see that $Q_{I} \propto X_{I}$ at the attractor point, with the constant of proportionality determined by the constraint (16) on the scalar. We thus find the explicit result

$$
\frac{X_{I}^{\mathrm{ext}}}{\mathcal{V}^{2 / 3}}=\frac{Q_{I}}{\left(\frac{1}{3 !} C^{J K L} Q_{J} Q_{K} Q_{L}\right)^{1 / 3}},
$$

for the attractor values of the scalar fields in terms of the charges. As a side product we found

$$
\frac{Z_{e}^{\mathrm{ext}}}{\mathcal{V}^{1 / 3}}=3\left(\frac{1}{3 !} C^{J K L} Q_{J} Q_{K} Q_{L}\right)^{1 / 3},
$$

for the central charge at the extremum.

\subsection{A Closer Look at the Attractor Mechanism}

Before considering examples, we follow up on some of the important features of the attractor mechanism that we skipped in the preceding subsection: we introduce the black hole entropy, we discuss the interpretation of the central charge, and we present some details on the units.

\section{Black Hole Entropy}

Having determined the scalars $X^{I}$ in terms of the charges we can now express the central charge (31) in terms of charges alone. It turns out that for spherically symmetric black holes the resulting expression is in fact related to the entropy through the simple formula

$$
S=2 \pi \cdot \frac{\pi}{4 G_{5}} \cdot\left(\frac{1}{3 \mathcal{V}^{1 / 3}} Z_{e}^{\mathrm{ext}}\right)^{3 / 2}
$$

The simplest way to establish this relation is to inspect a few explicit black hole solutions and then take advantage of near horizon symmetries to extend the result to large orbits of black holes that are known only implicitly. The significance of the formula (411) is that it allows the determination of the black hole entropy without actually constructing the black hole geometry.

In view of the explicit expression (40) for the central charge at the extremum we find the explicit formula

$$
S=2 \pi \cdot \frac{\pi}{4 G_{5}} \cdot \sqrt{\frac{1}{3 !} C^{J K L} Q_{J} Q_{K} Q_{L}},
$$

for the black hole entropy of a spherically symmetric, supersymmetric black hole in five dimensions. 


\section{Interpretation of the Central Charge}

In the preceding subsection we introduced the central charge (31) rather formally, as the linear combination of charges that satisfies a monotonic flow. This characterization can be supplemented with a nice physical interpretation as follows. The eleven-dimensional origin of the gauge potential $A_{t}^{I}$ can be determined from the decomposition (17). It is a three-form with one index in the temporal direction and the other two within the Calabi-Yau, directed along a $(1,1)$-cycle of type $I$. Such a three-form is sourced by $M 2$-branes wrapped on the corresponding $(1,1)$-cycle which we have denoted $\Omega^{I}$. The volume of this cycle is precisely $X^{I}$, according to (3). Putting these facts together it is seen that the central charge (31) is the total volume of the wrapped cycles, with multiple wrappings encoded in the charge $Q_{I}$. We can interpret the underlying microscopics as a single $M 2$-brane wrapping some complicated cycle $\Omega$ within the Calabi-Yau which can be characterized in terms of a decomposition

$$
\Omega=Q_{I} \Omega^{I},
$$

on the canonical cycles $\Omega^{I}$. Then the central charge is identified with the mass of this $M 2$-brane, up to an overall factor of the tension.

There is yet another interpretation of the central charge which takes as starting point the $N=2$ supersymmetry algebra

$$
\left\{Q_{\alpha}^{A}, Q_{\beta}^{B}\right\}=2\left(\delta^{A B} P_{\mu}\left(\Gamma^{\mu}\right)_{\alpha \beta}+\delta_{\alpha \beta} \epsilon^{A B} Z_{e}\right),
$$

where $A, B=1,2$ distinguish the two supercharges. The last term on the right hand side (proportional to $Z_{e}$ ) is the central term. It is introduced from a purely algebraic point of view, as a term that commutes with all other generators of the algebra. The algebra is most usefully analyzed in the restframe where $P_{\mu}\left(\Gamma^{\mu}\right)_{\alpha \beta}=P_{0}\left(\Gamma^{0}\right)_{\alpha \beta}$. Consider a state that is annihilated by one or more of the supercharges $Q_{\alpha}^{A}$. Taking expectation value on both sides with respect to this states, and demanding positive norm of the state, we find the famous BPS inequality

$$
M=\left|P_{0}\right| \geq Z_{e},
$$

with the inequality saturated exactly when supersymmetry is preserved by the state. Supersymmetric black holes are BPS states and so their mass should agree with the algebraic central charge. In the preceding paragraph we showed that the mass agrees with the central charge introduced geometrically, so the alternate introductions of the central charge agree.

\section{Some Comments on Units and Normalizations}

Let us conclude this subsection with a few comments on units. It is standard to introduce the eleven dimensional Planck length through $\kappa_{11}^{2}=(2 \pi)^{7} l_{P}^{9}$. In this notation the five dimensional Newton's constant is 


$$
G_{5}=\frac{\pi}{4} \cdot \frac{\left(2 \pi l_{P}\right)^{6}}{\mathcal{V}} \cdot l_{P}^{3}
$$

and the $M 2$-brane tension is $\tau_{M 2}=\frac{1}{(2 \pi)^{2} \ell_{P}^{3}}$. The relation to standard string theory units are $l_{P}=g_{s}^{1 / 3} \sqrt{\alpha^{\prime}}$ and the radius of the M-theory circle is $R_{11}=$ $g_{s} \sqrt{\alpha^{\prime}}$. Now, the physical charges $Q_{I}$ were introduced in (29) as the constant of integration from Gauss' law, following standard practice in supergravity. Such physical charges are proportional to quantized charges $n_{I}$ according to

$$
Q_{I}=\left(\frac{\mathcal{V}}{\left(2 \pi l_{P}\right)^{6}}\right)^{-2 / 3} \cdot l_{P}^{2} \cdot n_{I}=\left(\frac{\pi}{4 G_{5}}\right)^{-2 / 3} n_{I} .
$$

The mass of the brane configuration is

$$
M=\tau_{M 2} X^{I} n_{I}=\frac{1}{l_{P}^{3}} \cdot \frac{\mathcal{V}}{\left(2 \pi l_{P}\right)^{6}} \cdot \frac{X^{I}}{\mathcal{V}^{1 / 3}} \cdot Q_{I}=\frac{\pi}{4 G_{5}} \cdot \frac{X^{I}}{\mathcal{V}^{1 / 3}} \cdot Q_{I} .
$$

The formulae (47.48) are the precise versions of the informal notions that the charge $Q_{I}$ counts the number of branes and that the central charge $Z_{e}$ agrees with the mass. We see that there are awkward constants of proportionality, which vanish in units where $G_{5}=\frac{\pi}{4}$ and the volumes of two-cycles are measured relative to $\mathcal{V}^{1 / 3}$. In this first lecture we will for the most part go through the trouble of keeping all units around, to make sure that it is clear where the various factors go. In later lectures we will revert to the simplified units. ${ }^{2}$ If needed, one can restore units by referring back to the simpler special cases.

\subsection{An Explicit Example}

We conclude this introductory lecture by working out a simple example explicitly. The example we consider is when the Calabi-Yau space is just a torus $C Y=T^{6}$. Strictly speaking a torus is not actually a Calabi-Yau space if by the latter we mean a space with exactly $S U(3)$ holonomy. The issue is that for M-theory on $T^{6}$ the effective five-dimensional theory has $N=8$ supersymmetry rather than $N=2$ supersymmetry as we have assumed. This means there are extra gravitino multiplets in the theory which we have not taken into account. However, these gravitino multiplets decouple from the black hole background and so it is consistent to ignore them, in much the same way that we already ignore the $N=2$ hypermultiplets. We can therefore use the formalism reviewed above without any change.

In the explicit example we will further assume that the metric on the torus is diagonal so that the Kähler form takes the product form

$$
J=i\left(X^{1} d z^{1} \wedge d \bar{z}^{1}+X^{2} d z^{2} \wedge d \bar{z}^{2}+X^{1} d z^{3} \wedge d \bar{z}^{3}\right) .
$$

\footnotetext{
${ }^{2}$ In fact, the supersymmetry algebra (44) was already simplified this way, to avoid overly heavy notation.
} 
Then the scalar fields $X^{I}$ with $I=1,2,3$ are just the volumes of each $T^{2}$ in the decomposition $T^{6}=\left(T^{2}\right)^{3}$. The only nonvanishing intersection numbers of these two-cycles are $C_{123}=1$ (and cyclic permutations). The constraint (9) on the scalars therefore takes the simple form

$$
X^{1} X^{2} X^{3}=\mathcal{V} .
$$

The volumes (5) of four-cycles on the torus are

$$
X_{1}=X^{2} X^{3}=\mathcal{V} / X^{1} \quad \text { (and cyclic permutations) . }
$$

\section{Attractor Behavior}

The central charge (31) for this example is

$$
Z_{e}=X^{1} Q_{1}+X^{2} Q_{2}+X^{3} Q_{3} .
$$

According to the extremization principle we can determine the scalar fields at the horizon by minimizing this expression over moduli space. The constraint (50) can be implemented by solving in terms of one of the $X^{I}$ 's and then extremizing (52) over the two remaining moduli. Alternatively, one can employ Lagrange multipliers. Either way the result for the scalars in terms of the charges is

$$
\frac{X_{1}^{\text {ext }}}{\mathcal{V}^{1 / 3}}=\left(\frac{Q_{1}^{2}}{Q_{2} Q_{3}}\right)^{1 / 3}=\frac{Q_{1}}{\left(Q_{1} Q_{2} Q_{3}\right)^{1 / 3}} \quad \text { (and cyclic permutations) } .
$$

These are the horizon values for the scalars predicted by the attractor mechanism. They agree with the general formula (39). Below we confirm these values in the explicit solutions.

At the attractor point (53) the three terms in the central charge (52) are identical. The central charge takes the value

$$
Z_{\text {ext }}=3\left(Q_{1} Q_{2} Q_{3}\right)^{1 / 3} .
$$

The black hole entropy (41) becomes

$$
S=2 \pi \cdot \frac{\pi}{4 G_{5}} \cdot\left(Q_{1} Q_{2} Q_{3}\right)^{1 / 2}=2 \pi\left(n_{1} n_{2} n_{3}\right)^{1 / 2} .
$$

This is the entropy computed using the attractor formalism, i.e. without explicit construction of the black hole geometry. At the risk of seeming heavy handed, we wrote (55) both in terms of the proper (dimensionful) charges $Q_{I}$ and also in terms of the quantized charges $n_{I}$.

The entropy formula (55) is rather famous so let us comment a little more on the relation to other work. The $M 2$-brane black hole considered here can be identified, after duality to type IIB theory, with the $D 1-D 5$ black hole 
considered by Strominger and Vafa [5]. In this duality frame two of the M2brane charges become the background D-branes and the third charge is the momentum $p$ along the D1-brane. Then [55] coincides with Cardy's formula

$$
S=2 \pi \sqrt{\frac{c h}{6}},
$$

where the central charge $c=6 N_{1} N_{5}$ for the CFT on the D-branes and $h=p$ for the energy of the excitations. In the present lectures we are primarily interested in macroscopic features of black holes and no further details on the microscopic theory will be needed. For more review on this consult e.g. [6].

\section{Explicit Construction of the Black Holes}

We can compare the results from the attractor computation with an explicit construction of the black hole. The standard form of the $M 2$-brane solution in eleven-dimensional supergravity is

$$
d s_{11}^{2}=H^{-2 / 3} d x_{\|}^{2}+H^{1 / 3} d x_{\perp}^{2} .
$$

Here the space parallel to the $M 2$-brane is

$$
d x_{\|}^{2}=-d t^{2}+d x_{1}^{2}+d x_{2}^{2},
$$

when the spatial directions of the $M 2$-brane have coordinates $x_{1}$ and $x_{2}$. The transverse space $d x_{\perp}^{2}$ is written similarly in terms of the remaining eight coordinates. The function $H$ can be any harmonic on the transverse space; the specific one needed in our example is given below.

The harmonic function rule states that composite solutions can be formed by superimposing three $M 2$-brane solutions of the form (57) with cyclically permuted choices of parallel space. The only caveat is that we must smear along all directions within the torus, i.e. the harmonic functions can depend

only on the directions transverse to all the different branes. This procedure gives the standard intersecting $M 2$-brane solution

$$
d s_{11}^{2}=-f^{2} d t^{2}+f^{-1}\left(d r^{2}+r^{2} d \Omega_{3}^{2}\right)+\left[\left(\frac{H_{2} H_{3}}{H_{1}^{2}}\right)^{1 / 3}\left(d x_{1}^{2}+d x_{2}^{2}\right)+\text { cyclic }\right],
$$

where

$$
f=\left(H_{1} H_{2} H_{3}\right)^{-1 / 3} .
$$

We introduced radial coordinates in the four spatial dimensions transverse to all the branes. The harmonic functions are

$$
H_{I}=X_{I \infty}+\frac{Q_{I}}{r^{2}} \quad ; \quad I=1,2,3 .
$$


Comparing the intersecting brane solution (59) with the torus metric (49) we determine the scalar fields as

$$
\frac{X^{1}}{\mathcal{V}^{1 / 3}}=\left(\frac{H_{2} H_{3}}{H_{1}^{2}}\right)^{1 / 3} \quad \text { (and cyclic permutations) }
$$

The only remaining matter fields from the five-dimensional point of view are the gauge fields

$$
A^{I}=\partial_{r} H_{I}^{-1} d t \quad ; I=1,2,3 .
$$

The scalar fields $X^{I}$ (62) depend in a non-trivial way on the radial coordinate $r$. One can verify that the dependence is such that $Z_{e}=X^{I} Q_{I}$ is a monotonic function of the radii, but we will focus on the limiting values. The constants $X_{I \infty}$ in the harmonic functions (61) were introduced in order to obtain the correct limit as $r \rightarrow \infty$

$$
X^{1} \rightarrow\left(\frac{X_{2 \infty} X_{3 \infty}}{\left(X_{1 \infty}\right)^{2}}\right)^{1 / 3} \mathcal{V}^{1 / 3}=X_{\infty}^{1} \quad \text { (and cyclic permutations) }
$$

We used the constraint (50) in the asymptotic space and the relation (51) for the volumes of four-cycles. As the horizon $(r=0)$ is approached the moduli simplify to

$$
\frac{X^{1}}{\mathcal{V}^{1 / 3}} \rightarrow \frac{X_{\mathrm{hor}}^{1}}{\mathcal{V}^{1 / 3}}=\left(\frac{Q_{2} Q_{3}}{Q_{1}^{2}}\right)^{1 / 3} \quad \text { (and cyclic permutations) }
$$

In view of (51) this agrees with the values (53) predicted by the attractor mechanism.

We can also compute the black hole entropy directly from the geometry (59). The horizon at $r=0$ corresponds to a three-sphere with finite radius $R=\left(Q_{1} Q_{2} Q_{3}\right)^{1 / 6}$. Since $V_{S^{3}}=2 \pi^{2}$ for a unit three-sphere this gives the black hole entropy

$$
S=\frac{A}{4 G_{5}}=\frac{1}{4 G_{5}} \cdot 2 \pi^{2} \cdot R^{3}=2 \pi\left(n_{1} n_{2} n_{3}\right)^{1 / 2} .
$$

This explicit result for the black hole entropy is in agreement with (55) computed from the attractor mechanism.

\section{Black Ring Attractors}

In this lecture we generalize the discussion of the attractor mechanism to a much larger class of stationary supersymmetric black solutions to the $N=2$ theory in five dimensions introduced in section (2.2). By giving up spherical symmetry and allowing for dipole charges we can discuss multi-center black holes, rotating black holes and, especially, black rings. 


\subsection{General Supersymmetric Solutions}

The most general supersymmetric metric with a time-like Killing vector is

$$
d s^{2}=-f^{2}(d t+\omega)^{2}+f^{-1} d s_{4}^{2},
$$

where

$$
d s_{4}^{2}=h_{m n} d x^{m} d x^{n},
$$

is the metric of a four-dimensional base space and $\omega$ is a one-form on that base space. In the simplest examples the base is just flat space, but generally it can be any hyper-Kähler manifold in four dimensions. The matter fields needed to support the solution are the field strengths $F^{I}=d A^{I}$ given by

$$
F^{I}=d\left(f X^{I}(d t+\omega)\right)+\Theta^{I},
$$

and the scalar fields $X^{I}$ satisfying the sourced harmonic equation

$$
{ }^{(4)} \nabla^{2}\left(f^{-1} X_{I}\right)=\frac{1}{4} C_{I J K} \Theta^{J} \cdot \Theta^{K},
$$

on the base space. In these equations $\Theta^{I}$ is a closed self-dual two-form $\Theta^{I}=$ ${ }^{*_{4}} \Theta^{I}$ on the base. This two-form vanishes in the most familiar solutions but in general it must be turned on. For example, it plays a central role for black rings. The inner product between two-forms is defined as the contraction

$$
\alpha \cdot \beta=\frac{1}{2} \alpha_{m n} \beta^{m n} .
$$

The self-dual part of the one-form $\omega$ introduced in the metric (67) is sourced by $\Theta^{I}$ according to

$$
d \omega+{ }^{*_{4}} d \omega=-f^{-1} X_{I} \Theta^{I} .
$$

The general solution specified by equations $(67.72)$ is a bit impenetrable at first sight but things will become clearer as we study these equations. At this point we just remark that the form of the solution given above has reduced the full set of Einstein's equation and matter equations to a series of equations that are linear, if solved in the right order: first specify the hyper-Kähler base (68) and choose a self-dual two form $\Theta^{I}$ on that base. Then solve (70) for $f^{-1} X_{I}$. Determine the conformal factor $f$ of the metric from the constraint (9) and compute $\omega$ by solving (72). Finally the field strength is given in (69)

\footnotetext{
${ }^{3}$ We need $X^{I}$ which can be determined from (5). On a general Calabi-Yau this is a nonlinear equation, albeit an algebraic one.
} 


\subsection{The Attractor Mechanism Revisited}

We next want to generalize the discussion of the attractor mechanism from the spherical case considered in section (2.3) to the more general solutions described above. Thus we consider the gaugino variations

$$
\begin{aligned}
\delta \lambda_{i} & =\frac{i}{2} G_{I J} \partial_{i} X^{I}\left[\frac{i}{2} F_{\mu \nu}^{J} \Gamma^{\mu \nu}-\partial_{\mu} X^{J} \Gamma^{\mu}\right] \epsilon, \\
& =\frac{i}{2} G_{I J} \partial_{i} X^{I}\left[F_{m \hat{t}}^{J} \Gamma^{m}+\frac{i}{2} F_{m n}^{J} \Gamma^{m n}-\partial_{m} X^{J} \Gamma^{m}\right] \epsilon .
\end{aligned}
$$

In the second equation we imposed the supersymmetry projection (22) on the spinor $\epsilon$. In contrast to the spherically symmetric case (23) there are in general both electric $E_{m}^{I} \equiv F_{m \hat{t}}^{I}$ and magnetic $B_{m n}^{I} \equiv F_{m n}^{I}$ components of the field strength. However, as we explain below, it turns out that the magnetic field in fact does not contribute to (74). Therefore we have

$$
\frac{i}{2} G_{I J} \partial_{i} X^{I}\left[E_{m}^{J}-\partial_{m} X^{J}\right] \Gamma^{m} \epsilon=0 .
$$

Since this is valid for all components of $\epsilon$ we find

$$
G_{I J} \partial_{i} X^{I}\left[E_{m}^{J}-\partial_{m} X^{J}\right]=0
$$

just like (24) for the spherical symmetric case. In particular, we see that the gradient of the scalar field is related to the electric field quite generally. Of course this can be seen already from the explicit form (69) of the field strength, which can be written in components as

$$
\begin{aligned}
E_{m}^{I} & \equiv F_{m \hat{t}}^{I}=f^{-1} \partial_{m}\left(f X^{I}\right), \\
B_{m n}^{I} & \equiv F_{m n}^{I}=f X^{I}(d \omega)_{m n}+\Theta_{m n}^{I} .
\end{aligned}
$$

The point here is that we see how the relation (77) between the electric field and the gradient of scalars captures an important part of the attractor mechanism even when spherical symmetry is given up.

The key ingredient in reaching this result was the claim that the magnetic part (78) does not contribute to the supersymmetry variation (74). It is worth explaining in more detail how this comes about. The first term in (78) is of the form $F_{m n}^{I} \propto X^{I}(d \omega)_{m n}$. This term cancels from (174) because

$$
G_{I J} \partial_{i} X^{I} X^{J}=0
$$

due to special geometry. Let us prove this. Lowering the index using the metric (15) we can use (5) to find

$$
X_{I} \partial_{i} X^{I}=\frac{1}{2} C_{I J K} X^{J} X^{K} \partial_{i} X^{I}=\frac{1}{3 !} \partial_{i}\left(C_{I J K} X^{I} X^{J} X^{K}\right)=0 .
$$


due to the constraint (9) on the scalars $X^{I}$. This is what we wanted to show.

We still need to consider the second term in (78), the one taking the form $F_{m n}^{I} \propto \Theta_{m n}^{I}$. This term cancels from the supersymmetry variation (174) because the supersymmetry projection (22) combines with self-duality of $\Theta_{m n}^{I}$ to give

$$
\Theta_{m n}^{I} \Gamma^{m n} \epsilon=0 .
$$

In order to verify this recall that the $S O(4,1)$ spinor representation can be constructed from the more familiar $S O(3,1)$ spinor representation by including Lorentz generators from using the chiral matrix $\Gamma^{4} \equiv \gamma^{5}=-i \gamma^{0} \gamma^{1} \gamma^{2} \gamma^{3}$. All spinors that survive the supersymmetry projection (22) therefore satisfy $\Gamma^{1234} \epsilon=\epsilon$ by construction and this means the $\Theta_{12}$ term in (81) cancels the $\Theta_{34}$ term, etc.

After this somewhat lengthy and technical aside we return to analyzing the conditions (76). Following the experience from the spherically symmetric case we would like to trade the electric field for the charges, by using Gauss' law. The Lagrangean (11) gives the Maxwell equation

$$
d\left(G_{I J}{ }^{*} F^{J}\right)=\frac{1}{2} C_{I J K} F^{J} \wedge F^{K},
$$

with the source on the right hand side arising from the Chern-Simons term. Considering the coefficient of the purely spatial four-form we find Gauss' law

$$
\nabla^{m}\left(f^{-1} E_{m I}\right)=-\frac{1}{8} C_{I J K} \Theta^{J} \cdot \Theta^{K} .
$$

In arriving at this result we must take into account off-diagonal terms in the metric (67) due to the shift by $\omega$ of the usual time element $d t$. These contributions cancel with the terms coming from the first term in the field strength (78). Effectively this means only the term of the form $F_{m n}^{I} \sim \Theta_{m n}^{I}$ remains and it is those terms that give rise to the inhomogenous terms in (83). The physical interpretation is that the electric field is sourced by a distributed magnetic field which we may interpret as a delocalized charge density.

We are now ready to derive the generalized flow equation. Multiplying (76) by $\partial_{n} \phi^{i}$ and contract with the base metric $h^{m n}$ we find

$$
\partial^{m} X^{I} E_{m I}=G_{I J} \partial^{m} X^{I} \partial_{m} X^{J},
$$

which can be reorganized as

$$
\nabla^{m}\left(X^{I} f^{-1} E_{m I}\right)-X^{I} \nabla^{m}\left(f^{-1} E_{m I}\right)=f^{-1} G_{I J} \partial^{m} X^{I} \partial_{m} X^{J},
$$

and then Gauss' law (83) gives

$$
\nabla^{m}\left(X^{I} f^{-1} E_{m I}\right)=f^{-1} G_{I J} \partial^{m} X^{I} \partial_{m} X^{J}-\frac{X^{I}}{8} C_{I J K} \Theta^{J} \cdot \Theta^{K}
$$


This is the generalized flow equation. In the case where $\Theta^{I}=0$ the right hand side is positive definite and then the flow equation generalizes the monotonicity property found in (30) to many cases without radial symmetry. However, the most general case has nonvanishing $\Theta^{I}$ and such general flows are more complicated.

\subsection{Charges}

In order to characterize the more general flows with precision, it is useful to be more precise about how charges are defined.

Consider some bounded spatial region $V$. It is natural to define the electric charge in the region by integrating the electric flux through the boundary $\partial V$ as

$$
Q_{I}(V)=\frac{1}{2 \pi^{2}} \int_{\partial V} d S f^{-1} n^{m} E_{m I},
$$

where $n^{m}$ is an outward pointing normal on the boundary. If we consider two nested regions $V_{2} \subset V_{1}$ we have

$$
Q_{I}\left(V_{1}\right)-Q_{I}\left(V_{2}\right)=-\frac{1}{16 \pi^{2}} \int d^{4} x \sqrt{h} C_{I J K} \Theta^{J} \cdot \Theta^{K},
$$

where the second step used Gauss' law (83). This means the charge is monotonically decreasing as we move to larger volumes. The reason that it does not have to be constant is that in general the delocalized source on the right hand side of (83) contributes.

The central charge is constructed from the electric charges by dressing them with the scalar fields. It was originally introduced in (31) but, in analogy with the definition (87) of the electric charge in a volume of space, we may dress the electric field by the scalars as well and so introduce the central charge in a volume of space as

$$
Z_{e}(V)=\frac{1}{2 \pi^{2}} \int_{\partial V} d S f^{-1} n^{m} X^{I} E_{m I} .
$$

Considering again a nested set of regions we can use t86 the show the central charge satisfies

$Z_{e}\left(V_{1}\right)-Z_{e}\left(V_{2}\right)=\frac{1}{2 \pi^{2}} \int d^{4} x \sqrt{h}\left[f^{-1} G_{I J} \nabla^{m} X^{I} \nabla_{m} X^{J}-\frac{1}{8} C_{I J K} X^{I} \Theta^{J} \cdot \Theta^{K}\right]$.

When $\Theta^{I}=0$ the central charge is monotonically increasing as we move outwards. This generalizes the result from the spherically symmetric case to all cases where the two-forms vanish. When the system is not spherically symmetric there is no unique "radius" but this is circumvented by the introduction of nested regions, which gives an orderly sense of moving "outwards". Note that 
in general we do not force the nested volumes to preserve topology. In particular there can be multiple singular points and these then provide natural centers of the successive nesting.

When the two-forms $\Theta^{I} \neq 0$ the electric central charge (89) may not be monotonic and the flow equation does not provide any strong constraint on the flow.

In order to interpret the $\Theta^{I}$ 's properly we would like to associate charges with them as well. Since they are two-forms it is natural to integrate them over two-spheres and so define

$$
q^{I}=-\frac{1}{2 \pi} \int_{S^{2}} \Theta^{I} .
$$

Since the two-forms $\Theta^{I}$ are closed the integral is independent under deformations of the two-cycle and, in particular, it vanishes unless the $S^{2}$ is noncontractible on the base space. One way such non-trivial cycles can arise is by considering non-trivial base spaces. For our purposes the main example will be when the base space is flat, but endowed with singularities along one or more closed curves (including lines going off to infinity). This situation also gives rise to noncontractible $S^{2}$ 's because in four Euclidean dimensions a line can be wrapped by surfaces that are topologically a two-sphere.

The charges $q^{I}$ defined in (91) can be usefully thought as a magnetic charges. In our main example of a flat base space with a closed curve we may interpret the configuration concretely in terms of electric charge distributed along the curve. Since the curve is closed there is in general no net electric charge, but there will be a dipole charge and it is this dipole charge that we identify as the magnetic charge (91).

In keeping with the analogy between the electric and magnetic charges we would also like to introduce a magnetic central charge. The electric central charge (89) was obtained by dressing the ordinary charge (87) by the moduli. In analogy, we construct the magnetic central charge

$$
Z_{m}(V)=-\frac{1}{2 \pi} \int_{S^{2}} X_{I} \Theta^{I} .
$$

In some examples this magnetic central charge will play a role analogous to that played by the electric central charge in the attractor mechanism.

A general configuration can be described in terms of its singularities on the base space. There may be a number of isolated point-like singularities, to which we assign electric charges, and there may be a number of closed curves (including lines going off to infinity), to which we assign magnetic charges.

In four dimensions electric and magnetic charges are very similar: they are related by electric magnetic duality, which is implemented by symplectic transformations in the complex special geometry. In five dimensions the situation is more complicated because the Chern-Simons term makes the symmetry between point-like electric sources and string-like magnetic sources more subtle. Therefore we will need to treat them independently. 


\subsection{Near Horizon Enhancement of Supersymmetry}

There is another aspect of attractor behavior that we have not yet developed: the attractor leads to enhancement of supersymmetry 7 . This is a very strong condition that completely determines the attractor behavior, even when dipole charges are turned on.

The enhancement of supersymmetry means the entire supersymmetry of the theory is preserved near the horizon. To appreciate why that is such a strong conditions, recall the origin of the attractor flow: we considered the gaugino variation (74) and found the flow by demanding that the various terms cancel. The enhancement of supersymmetry at the attractor means each term vanishes by itself.

We first determine the supersymmetry constraint on the gravitino variation (20). By considering the commutator of two variations [7, it can be shown that the near horizon geometry must take the form $A d S_{p} \times S^{q}$. In five dimensions there are just two options: $A d S_{3} \times S^{2}$ or $A d S_{2} \times S^{3}$.

The near horizon geometry of the supersymmetric black hole in five dimension that we considered in section (2.5) is indeed $A d S_{2} \times S^{3}$ [8] (up to global identifications). A more stringent test is the attractor behavior of the supersymmetric rotating black hole. One might have expected that rotation would squeeze the sphere and make it oblate but this would not be consistent with enhancement of supersymmetry. In fact, it turns out that, for supersymmeric black holes, the near horizon geometry indeed remains $A d S_{2} \times S^{3}[9$ (up to global identifications).

There are also examples of a supersymmetric configurations with near horizon geometry $A d S_{3} \times S^{2}$. The simplest example is the black string in five dimensions. A more general solution is the supersymmetric black ring, which also has near horizon geometry $A d S_{3} \times S^{2}$. Indeed, the extrinsic curvature of the ring becomes negligible in the very near horizon geometry so there the black ring reduces to the black string. We will consider these examples in more detail in the next section.

The pattern that emerges from these examples is that black holes correspond to point-like singularities on the base and a near horizon geometry $A d S_{2} \times S^{3}$ in the complete space. On the other hand, black strings and black rings correspond to singularities on a curve in the base and a near horizon geometry $A d S_{3} \times S^{2}$ in the complete space. The two classes of examples are related by electric-magnetic duality which, in five dimensions, interchanges one-form potentials with two-form potentials and so interchanges black holes and black strings. This duality interchanges $A d S_{3} \times S^{2}$ with $A d S_{2} \times S^{3}$.

So far we have just considered the constraints from the gravitino variation (20). The attractor behavior of the scalars is controlled by the gaugino variation (74) which we repeat for ease of reference

$$
\delta \lambda_{i}=\frac{i}{2} G_{I J} \partial_{i} X^{I}\left[F_{m \hat{t}}^{J} \Gamma^{m \hat{t}}+\frac{i}{2} F_{m n}^{J} \Gamma^{m n}-\partial_{m} X^{J} \Gamma^{m}\right] \epsilon .
$$


Near horizon enhancement of supersymmetry demands that each term in this equation vanishes by itself, since no cancellations are possible when the spinor $\epsilon$ remains general. Let us consider the three conditions in turn.

The vanishing of the third term $\partial_{m} X^{J}=0$ means $X^{J}$ is a constant in the near horizon geometry. The attractor mechanism will determine the value of that constant as a function of the charges.

The first term in (93) reads

$$
\partial_{i} X^{I} E_{I m}=0
$$

in terms of the electric field introduced in (77). In the event that there is a point-like singularity in the base space there is an $S^{3}$ in the near horizon geometry. Integrating the flux over this $S^{3}$ and recalling the definition (87) of the electric charge we then find

$$
\partial_{i} Z_{e}=0
$$

in terms of the electric central charge (31). This is the attractor formula (36), now applicable in the near any point-like singularity in base space. We can readily determine the explicit attractor behavior as (39) near any horizon with $S^{3}$ topology.

We did not yet consider the condition that the second term in (93) vanishes. This term was considered in some detail after (78). There we found that the magnetic field $B_{m n}^{I}=F_{m n}^{I}$ has a term proportional to $X^{I}$ which cancels automatically from the supersymmetry conditions, due to special geometry relations. However, there is also another term in $B_{m n}^{I}$ which is proportional to $\Theta_{m n}^{I}$. This term also cancels from the supersymmetry variation, but only for the components of the supersymmetry generator $\epsilon$ that satisfy the projection (22). However, in the near horizon region there is enhancement of supersymmetry and so the variation must vanish for all components $\epsilon$. This can happen if the two-forms $\Theta^{I}$ take the special form

$$
\Theta^{I}=k X^{I},
$$

where $k$ is a constant ( $I$-independent) two-form because then special geometry relations will again guarantee supersymmetry. The special form 96 will determine the scalars completely.

Indeed, suppose that sources are distributed along a curve in the base space. Then we can integrate (96) along the $S^{2}$ wrapping the curve. This gives

$$
q^{I}=-\frac{1}{2 \pi} \int_{S^{2}} \Theta^{I}=X_{\mathrm{ext}}^{I} \cdot \text { constant },
$$

for the dipole charges in the near horizon region. The constant of proportionality is determined by the constraint (9) and so we reach the final result ${ }^{4}$

\footnotetext{
${ }^{4}$ In this sections 3 and 4 we use the simplified units where $\mathcal{V}=1$ and $G_{5}=\frac{\pi}{4}$. See section 2.4 for details on units.
} 


$$
X_{\mathrm{ext}}^{I}=\frac{q^{I}}{\left(\frac{1}{3 !} C_{J K L} q^{J} q^{K} q^{L}\right)^{1 / 3}},
$$

for the scalar field in terms of the dipole charges. The result is applicable near singularities distributed along a curve in the base space. In particular, this is the attractor value for the scalars in the near horizon region of black strings and of black rings.

Our result (98) was determined directly in the near horizon region, by exploiting the enhancement of supersymmetry there. In the case where $\Theta^{I} \neq 0$ we cannot understand the entire flow as a gradient flow of the electric central charge $Z_{e}$, nor are the attractor values given by extremizing $Z_{e}$. In fact, we can see that the attractor values (98) amount to extremization of the magnetic central charge (92). However, the significance of this is not so clear since it is only the near horizon behavior that is controlled by $Z_{m}$, not the entire flow. It would be interesting to find a more complete description of the entire flow in the most general case. For now we understand the complete flow when $\Theta^{I}=0$, and the attractor behavior when $\Theta^{I} \neq 0$.

There is in fact another caveat we have not mentioned so far. Our expression (39) for the scalars at the electric attractor breaks down when $C^{J K L} Q_{J} Q_{K} Q_{L}=0$, and similarly (98) for the magnetic attractor breaks down when $C_{I J K} q^{I} q^{J} q^{K}=0$. In the electric case the issue has been much studied: the case where $C^{J K L} Q_{J} Q_{K} Q_{L}=0$ corresponds to black holes with area that vanishes classically. These are the small black holes. In some cases it is understood how higher derivative corrections to the action modify the attractor behavior such that the geometry and the attractor values of the scalars become regular 11, 12. The corresponding magnetic case $C_{I J K} q^{I} q^{J} q^{K}=0$ corresponds to small black rings. This case has been studied less but it is possible that a similar picture applies in that situation.

\subsection{Explicit Examples}

In this subsection we consider a number of explicit geometries. In each example we first determine the attractor behavior abstractly, by applying the attractor mechanism, and then check the results by inspecting the geometry.

\section{The Rotating Supersymmetric Black Hole}

The simplest example of the attractor mechanism is the spherically symmetric black hole discussed in detail in section (2.5). The generalization of the spherically symmetric solution to include angular momentum are the rotating supersymmetric black hole in five-dimensions. This solution is known as the BMPV black hole [18.

Let us consider the attractor mechanism first. The rotating black hole is electrically charged but there are no magnetic charges, so the two-forms $\Theta^{I}$ vanish in this case. We showed in section (3.2) that then the electric 
central charge $Z_{e}$ must be monotonic just as it was in the nonrotating case. Extremizing over moduli space we therefore return to the values (53) of the scalars found in the non-rotating case. Alternatively we can go immediately to the general result (39) which is written for a general Calabi-Yau three-fold. Either way, we see that the attractor values of the scalars are independent of the black hole angular momentum. Since the rotation deforms the black hole geometry, this result is not at all obvious. The independence of angular momentum is a prediction of the attractor mechanism.

We can verify the result by inspecting the explicit black hole solution. The metric takes the form (67) where the base space $d x_{4}^{2}$ is just flat space $R^{4}$ which can be written in spherical coordinates as

$$
d x_{4}^{2}=d r^{2}+r^{2}\left(d \theta^{2}+\cos ^{2} \theta d \psi^{2}+\sin ^{2} \theta d \phi^{2}\right) .
$$

Although the solution is rotating, it is almost identical to the non-rotating example discussed in section (2.5): the conformal factor $f$ is given again by (60) where the harmonic functions $H_{I}$ are given by (61). Additionally, the matter fields remain the scalar fields (62) and the gauge fields (63). The only effect of adding rotation is that now the one-forms $\omega$ are

$$
\omega=-\frac{J}{r^{2}}\left(\cos ^{2} \theta d \phi+\sin ^{2} \theta d \psi\right) .
$$

As an aside we note that the self-dual part of $d \omega$ vanishes, as it must for solutions with $\Theta^{I}=0$, but the anti-selfdual part is non-trivial: it carries the angular momentum.

Now, for the purpose of the attractor mechanism we are especially interested in the scalar fields. As just mentioned, these take the form (62) in terms of the harmonic functions, independently of the angular momentum. This means they will in fact approach the attractor values (53) at the horizon. In particular, the result is independent of the angular momentum, as predicted by the attractor mechanism.

\section{Multi-center black holes}

From the supergravity point of view, the $M 2$-brane solution (57) is valid for any harmonic function $H$ on the transverse space. Similarly, the intersecting brane solution (59) (and its generalization to an arbitrary Calabi-Yau threefold) remains valid for more general harmonic functions $H_{I}$. In particular, the standard harmonic functions (61) can be replaced by

$$
H_{I}=X_{I \infty}+\sum_{i=1}^{N} \frac{Q_{I}^{(i)}}{\left|\boldsymbol{r}-\boldsymbol{r}_{i}\right|^{2}}
$$

where $\boldsymbol{r}_{i}$ are position vectors in the transverse space. We will assume that all $Q_{I}^{(i)}>0$ so that the configuration is regular. 
The interpretation of these more general solutions is that they correspond to multi-center black holes, i.e. $N$ black holes coexisting in equilibrium, with their gravitational attraction cancelled by repulsion of the charges. The black hole centered at $\boldsymbol{r}_{i}$ has charges $\left\{Q_{I}^{(i)}\right\}$.

The attractor behavior of these solutions is the obvious generalization of the single center black holes. The attractor close to each center depends only on the charges associated with that center, because the charge integrals 877) are defined with respect to singularities on the base manifolds. This immediately implies that the attractor values for the scalars in a particular attractor region are (39) in terms of the charges $\left\{Q_{I}^{(i)}\right\}$ associated with this particular region.

The explicit solutions verify this prediction of the attractor mechanism because the harmonic functions (101) are dominated by the term corresponding to a single center in the attractor regime corresponding to that center.

In some ways the multi-center solution is thus a rather trivial extension of the single-center solution. The reason it is nevertheless an interesting and important example is the following. Far from all the black holes, the geometry of the multi-center black hole approaches that of a single center solution with charges $\left\{Q_{I}\right\}=\left\{\sum_{i=1}^{N} Q_{I}^{(i)}\right\}$. Based on the asymptotic data alone one might have expected an attractor flow governed by the corresponding central charge $Z_{e}=X^{I} Q_{I}$, leading to the attractor values for the scalars depending on the $Q_{I}$ in a unique fashion, independently of the partition of the geometry into constituent black holes with charges $\left\{Q_{I}^{(i)}\right\}$. The multi-center black hole demonstrates that this expectation is false: the asymptotic behavior does not uniquely specify the attractor values of the scalars, and nor does it define the near horizon geometry and the entropy.

More structure appears when one goes beyond the focus on attractor behavior and consider the full attractor flow of the scalars. As we discussed in section (3.2), the flow of the scalars is a gradient flow controlled by the electric central charge (this is when the dipoles vanish). The central charge is interpreted as the total constituent mass. For generic values of the scalar fields the actual mass of the configuration is smaller, i.e. the black holes are genuine bound states. Now, in the course of the attractor flow, the values of the scalars change. At some intermediate point it may be that the actual mass of the black hole is identical to that of two (or more) clusters of constituents. This is the point of marginal stability. There the attractor flow will split up, and continue as several independent flows, each controlled by the appropriate sets of smaller charges. This process then continues until the true attractor basins are reached. The total flow is referred to as the split attractor flow. It has interesting features which are beyond the scope of the present lecture. We refer the reader to the original papers [10] and the review [3].

\section{Supersymmetric Black Strings}

The black string is a five dimensional solution that takes the form 


$$
d s_{5}^{2}=f^{-1}\left(-d t^{2}+d x_{4}^{2}\right)+f^{2}\left(d r^{2}+r^{2} d \Omega_{2}^{2}\right),
$$

where the conformal factor

$$
f=\frac{1}{3 !} C_{I J K} H^{I} H^{J} H^{K},
$$

in terms of the harmonic function

$$
H^{I}=X_{\infty}^{I}+\frac{q^{I}}{2 r} .
$$

The geometry is supported by the gauge fields

$$
A^{I}=-\frac{1}{2} q^{I}(1+\cos \theta) d \phi,
$$

and the scalar fields

$$
X^{I}=f^{-1} H^{I} .
$$

The black string solution is the long distance representation of an M5brane that wraps the four-cycle $q^{I} \Omega_{I}$ inside a Calabi-Yau threefold and has the remaining spatial direction aligned with the coordinate $x^{4}$. This configuration plays in important role in microscopic considerations of the four dimensional black hole (see e.g. [13]).

The gauge field (105) corresponds to the field strength $F^{I}=-q^{I} \sin \theta d \theta d \phi$. This is a magnetic field, with normalization of the charge in agreement with the one introduced in (91). The black string is therefore an example where the two-forms $\Theta^{I} \neq 0$.

We should note that the metric (102) of the supersymmetric black string differs from the form (67), assumed in the analysis in this lecture. The reason that a different form of the metric applies is that the black string has a null Killing vector whereas (67) assumes a time-like Killing vector. Nevertheless, we can think of the null case as a limiting case of the time-like one. Concretely, if there is a closed curve on the base-space of (67), the black string is the limit where the curve is deformed such that two points are taken to infinity and only a straight line remains (i.e. the return line is fully at infinity). This limiting procedure is how the simple black string arises from the more complicated black ring solution (see following example).

Let us now examine the attractor behavior of the black string. In section (3.4) we showed that near horizon enhancement of supersymmetry demands that, at the attractor, the two forms simplify to $\Theta^{I}=k X^{I}$ where $k$ is a constant ( $I$ independent) two-form. This condition was then showed to imply the expression (98) for the scalars as functions of the magnetic charges.

We can verify the attractor behavior by inspection of the explicit solution. Taking the limit $r \rightarrow 0$ on the scalars (106) we find

$$
X_{\text {hor }}^{I}=\frac{q^{I}}{\left(\frac{1}{3 !} C_{J K L} q^{J} q^{K} q^{L}\right)^{1 / 3}} .
$$

This agrees with (98) predicted by the attractor mechanism. 


\section{Black Rings}

As the final example we consider the attractor behavior near the supersymmetric black ring [19, 20]. This is a much more involved example which in fact was the motivation for the development of the formalism considered in this lecture.

The supersymmetric black ring is charged with respect to both electric charges $Q_{I}$ and dipole charges $q^{I}$. Far from the ring the geometry is dominated by the electric charges, which have the slowest asymptotic fall-off, and the value of the charges can be determined using Gauss' law (87). The dipole charges are determined according to (91) where the by $S^{2}$ is wrapped around the ring. Since the two-forms do not vanish they dominate the near horizon geometry and the near horizon values of the scalar fields become (98), as they were for the black string.

We can verify the result from the attractor mechanism by inspecting the explicit black ring solution. The metric takes the general form (67). The conformal factor $f$ is given by (60) in terms of functions $H_{I}$ which take the form:

$$
H_{I}=X_{I \infty}+\frac{Q_{I}-\frac{1}{2} C_{I J K} q^{J} q^{K}}{\Sigma}+\frac{1}{2} C_{I J K} q^{J} q^{K} \frac{r^{2}}{\Sigma^{2}},
$$

where

$$
\Sigma=\sqrt{\left(r^{2}-R^{2}\right)^{2}+4 R^{2} r^{2} \cos ^{2} \theta} .
$$

Although $H_{I}$ play the same role as the harmonic functions in other examples they are in fact not harmonic: they satisfy equations with sources. The expression for $\Sigma$ vanishes when $r=R, \theta=\frac{\pi}{2}$, and arbitrary $\psi$. Therefore the functions $H_{I}$ diverge along a circle of radius $R$ in the base space. This is the ring.

The full solution in five dimension remains regular, due to the conformal factor. At large distances $H_{I} \sim X_{I \infty}+\frac{Q_{I}}{r^{2}}$ so the black ring has the same asymptotic behavior as the spherically symmetric black hole considered in section (2.5). This is because the dipole charges die off asymptotically and so $H_{I}$ differ from that of a black hole only at order $\mathcal{O}\left(\frac{1}{r^{4}}\right)$. However, the dipole charges dominate close to the horizon.

The scalar fields in the supersymmetric black ring solution take the form

$$
X_{I}=\frac{H_{I}}{\left(\frac{1}{3 !} C^{J K L} H_{J} H_{K} H_{L}\right)^{1 / 3}} .
$$

In the near horizon region where the "harmonic" functions $H_{I}$ diverge the scalars approach

$$
X^{I}=\frac{q^{I}}{\left(\frac{1}{3 !} C_{J K L} q^{J} q^{K} q^{L}\right)^{1 / 3}} .
$$

This is in agreement with the prediction (98) from the attractor mechanism.

In the preceding we defined just enough of the black ring geometry to consider the attractor mechanism. For completeness, let us discuss also the 
remaining features. They are most conveniently introduced in terms of the ring coordinates

$$
h_{m n} d x^{m} d x^{n}=\frac{R^{2}}{(x-y)^{2}}\left[\frac{d y^{2}}{y^{2}-1}+\left(y^{2}-1\right) d \psi^{2}+\frac{d x^{2}}{1-x^{2}}+\left(1-x^{2}\right) d \phi^{2}\right],
$$

on the base space. Roughly speaking, the $x$ coordinate is a polar angle $x \sim$ $\cos \theta$ that combines with $\phi$ to form two-spheres in the geometry. The angle along the ring is $\psi$, and $y$ can be interpreted as a radial direction with $y \rightarrow-\infty$ at the horizon. In terms of these coordinates the two form sources are

$$
\Theta^{I}=-\frac{1}{2} q^{I}(d y \wedge d \psi+d x \wedge d \phi) .
$$

Integrating the expression along the $S^{2}$,s we can verify that the normalization agrees with the definition (91) of magnetic charges.

The final element of the geometry is the one-form $\omega$ introduced in (67). Its nonvanishing components are

$$
\begin{aligned}
& \omega_{\psi}=-\frac{1}{R^{2}}\left(1-x^{2}\right)\left[Q_{I} q^{I}-\frac{1}{6} C_{I J K} q^{I} q^{J} q^{K}(3+x+y)\right], \\
& \omega_{\phi}=\frac{1}{2} X_{I \infty} q^{I}(1+y)+\omega_{\psi} .
\end{aligned}
$$

In five dimensions there are two independent angular momenta which we can choose as $J_{\phi}$ and $J_{\psi}$. The one form (114 115) gives their values as

$$
\begin{aligned}
J_{\phi} & =\frac{\pi}{8 G_{5}}\left(Q_{I} q^{I}-\frac{1}{6} C_{I J K} q^{I} q^{J} q^{K}\right), \\
J_{\psi} & =\frac{\pi}{8 G_{5}}\left(2 R^{2} X_{I \infty} q^{I}+Q_{I} q^{I}-\frac{1}{6} C_{I J K} q^{I} q^{J} q^{K}\right) .
\end{aligned}
$$

These expressions will play a role in the discussion of the interpretation of the attractor mechanism in the next section.

\section{Extremization Principles}

An alternative approach to the attractor mechanism is to analyze the Lagrangian directly, without using supersymmetry 14. An advantage of this method is that the results apply to all extremal black holes, not just the supersymmetric ones [15]. A related issue is the understanding of the attractor mechanism in terms of the extremization of various physical quantities.

\subsection{The Reduced Lagrangian}

The attractor mechanism can be analyzed without appealing to supersymetry, by starting directly from the Lagrangian. In this subsection we exhibit the details. 
We will consider just the spherically symmetric case with the metric

$$
d s^{2}=-f^{2} d t^{2}+f^{-1}\left(d r^{2}+r^{2} d \Omega_{3}^{2}\right) .
$$

Having assumed spherical symmetry, it follows that the gauge field strengths take the form (29). The next step is to insert the ansatz into the Lagrangian (111). The result will be a reduced Lagrangian that depends only on the radial variable. In order to advantage of intuition from elementary mechanics, it is useful to trade the radial coordinate for an auxiliary time coordinate defined by

$$
d r=-\frac{1}{2} r^{3} d \tau \quad ; \quad \partial_{r}=-\frac{2}{r^{3}} \partial_{\tau} .
$$

Introducing the convenient notation

$$
f=e^{2 U},
$$

a bit of computation gives the reduced action

$$
L d \tau=\left[-6\left(\partial_{\tau} U\right)^{2}-G_{I J} \partial_{\tau} X^{I} \partial_{\tau} X^{J}+\frac{1}{4} e^{4 U} G^{I J} Q_{I} Q_{J}\right] d \tau,
$$

up to overall constants.

Imposing a specific ansatz on a dynamical system removes numerous degrees of freedom. The corresponding equations of motion appear as constraints on the reduced system. In the present setting the main issue is that the charges specified by the ansatz are the momenta conjugate to the gauge fields. The correct variational principle is then obtained by a Legendre transform which, in this simple case, simply changes the sign of the potential in (121). Thus the equations of motion of the reduced system can be obtained in the usual way from the effective Lagrangean

$$
\mathcal{L}=\left[-6\left(\partial_{\tau} U\right)^{2}-G_{I J} \partial_{\tau} X^{I} \partial_{\tau} X^{J}-\frac{1}{4} e^{4 U} G^{I J} Q_{I} Q_{J}\right] .
$$

It is instructive to rewrite the effective potential in (121) and (122). Using the relations (14 15) we can show the identity

$$
G^{I J} Q_{I} Q_{J}=\frac{2}{3} Z_{e}^{2}+G^{I J} D_{I} Z_{e} D_{J} Z_{e},
$$

where we used the definition (31) of the electric central charge $Z_{e}$ and (38) of the covariant derivative on moduli space. The Lagrangean (122) can be written as

$$
\begin{aligned}
\mathcal{L} & =-6\left(\partial_{\tau} U\right)^{2}-G_{I J} \partial_{\tau} X^{I} \partial_{\tau} X^{J}-\frac{1}{6} e^{4 U} Z_{e}^{2}-\frac{1}{4} e^{4 U} G^{I J} D_{I} Z_{e} D_{J} Z_{e} \\
& =-6\left(\partial_{\tau} U \pm \frac{1}{6} e^{2 U} Z_{e}\right)^{2} \\
& -G_{I J}\left(\partial_{\tau} X^{I} \pm \frac{1}{2} e^{2 U} G^{I K} D_{K} Z_{e}\right)\left(\partial_{\tau} X^{J} \pm \frac{1}{2} e^{2 U} G^{J L} D_{L} Z_{e}\right) \pm \partial_{\tau}\left(e^{2 U} Z_{e}\right)
\end{aligned}
$$


where we used ${ }^{5}$

$$
\partial_{\tau} X^{I} D_{I} Z_{e}=\partial_{\tau} Z_{e}
$$

Thus the Lagrangean can be written a sum of squares, up to a total derivative. We can therefore find extrema of the action by solving the linear equations of motion

$$
\begin{aligned}
\partial_{\tau} U & =-\frac{1}{6} e^{2 U} Z_{e}, \\
\partial_{\tau} X^{I} & =-\frac{1}{2} e^{2 U} G^{I J} D_{J} Z_{e} .
\end{aligned}
$$

The second equation is identical to the condition (35) that the gaugino variations vanish, as one can verify by identifying variables according to the various notations we have introduced. The first equation can be interpreted as the corresponding condition that the gravitino variation vanish. To summarize, we have recovered the conditions for supersymmetry by explicitly writing the bosonic Larangean as a sum of squares, so that extrema can be found by solving certain linear equations of motion. The analysis of these linear equations can now be repeated from section 2.3. In particular, finite energy density at the horizon (or enhancement of supersymmetry, as discussed in section 3.4) implies the conditions $D_{I} Z_{e}=0$, and these in turn lead to the explicit form (40) for the attractor values of the scalars.

One of the advantages of this approach to the attractor mechanism is that it applies even when supersymmetry is broken. To see this, consider solutions with constant value of the scalar fields throughout spacetime $\partial_{\tau} X^{I}=$ 0 . Extremizing the Lagrangian with respect to the scalar fields can then be found by considering just the potential (123). Upon variation we find

$$
\left(\frac{2}{3} G_{I J} Z_{e}+D_{I} D_{J} Z_{e}\right) D^{J} Z_{e}=0
$$

This equation is solved automatically for $D_{J} Z_{e}=0$. Such geometries are the supersymmetric solutions that have been our focus. However, it is seen that there can also be solutions where the scalars satisfy

$$
\frac{2}{3} G_{I J} Z_{e}+D_{I} D_{J} Z_{e}=0
$$

Such solutions do not preserve supersymmetry, but they do exhibit attractor behavior.

\subsection{Discussion: Physical Extremization Principles}

In section 3.4 we found that the attractor values are determined by extremizing one of the two central charges. For $\Theta^{I}=0$ they are determined by

\footnotetext{
${ }^{5}$ We can verify this by writing $D_{I} Z_{e}=\mathcal{V}^{1 / 3} \partial_{I}\left(\mathcal{V}^{-1 / 3} Z_{e}\right)$. This amounts to changing into physical coordinates before taking the derivative and then changing back.
} 
extremizing the electric central charge (31) over moduli space $\partial_{i} Z_{e}=0$. On the other hand, for $\Theta^{I} \neq 0$, we should instead extremize the magnetic central charge $\partial_{i} Z_{m}=0$. These prescriptions are mathematically precise but they lack a clear physical interpretation. It would be nice to reformulate the extremization principles in terms of physical quantities.

Let us consider first the situation when $\Theta^{I}=0$. As discussed in section [2.4 the electric central charge can be interpreted as the mass of the system. Therefore, extremization amounts to minimizing the mass. If we think about the attractor mechanism this way, the monotonic flow of the electric central charge amounts to a roll down a potential, with scalars ultimately taking the value corresponding to dynamical equilibrium. In particular, if the scalars are adjusted to their attractor values already at infinity (these configurations are referred to as "double extreme black holes") there is no flow because the configuration remains in its equilibirum.

A difficulty with this picture is the fact that the situation with $\Theta^{I} \neq 0$ works very differently even though the asymptotic configuration is in fact independent of the dipole charges. We would like a physical extremization principle that works for that case as well. The case where $\Theta^{I} \neq 0$ is elucidated by considering the combination

$$
J_{\psi}-J_{\phi}=R^{2} X_{I \infty} q^{I}=R^{2} Z_{m},
$$

of the angular momenta (116 117). This quantity can be interpreted as the intrinsic angular momentum of the black ring, not associated with the surrounding fields. The interesting point is that extremizing $Z_{m}$ is the same as extremizing $J_{\psi}-J_{\phi}$ with $R^{2}$ fixed. It may at first seem worrying that we propose extremizing angular momenta. For a black hole these would be quantum numbers measurable at infinity, and so they would be part of the input that specifies solution. However, the black ring solution is different: we can choose its independent parameters as $q^{I}, Q_{I}, R^{2}$ with the understanding that then the angular momenta $J_{\phi}$ and $J_{\psi}$ that support the black ring must be those determined by (116 117). The precise values of $J_{\phi}$ and $J_{\psi}$ so determined depend on the scalars and the proposed extremization principle is that the scalars at the horizon are such that the combination (131) is minimal.

The proposed principle is quite similar to the extremization of the mass in the electric case of supersymmetric black holes. In fact, the combination (131) of angular momenta that we propose extremizing in the magnetic case behaves very much like a mass: it can be interpreted as the momentum along the effective string that appears in the near ring limit [16, 17.

In order to elevate the extremization of (131) to a satisfying principle one would need a geometric definition of the ring radius $R$ that works independently of the explicit solution. Ideally, there should be some kind of conserved integral, akin to those defining the electric charges, or the more subtle ones appearing for dipole charges [21. Another issue is that of more complicated multiple ring solutions, which are characterized by several radii. This latter problem is completely analogous to the ambiguity with assigning mass for 
multi black hole solutions: the asymptotics does not uniquely specify the near horizon behavior. We will put these issues aside for now, and seek an extremization principle that combines the extremization of (131) in the magnetic case with extremization of the mass in electric case, and works in any basin of attraction, whether electric or magnetic in character.

To find such a principle, recall that the black hole entropy (41) can be written in terms of the central charge in the electric case. Accordingly, the extremization over moduli space can be recast as ${ }^{6}$

$$
\partial_{i} S=0
$$

The black ring entropy can be written compactly as

$$
S=2 \pi \sqrt{J_{4}} .
$$

For toroidal compactification ${ }^{7} J_{4}$ is the quartic $E_{7(7)}$ invariant, evaluated at arguments that depend on the black ring parameters according to the identifications

$$
J_{4}=J_{4}\left(Q_{I}, q^{I}, J_{\psi}-J_{\phi}\right) .
$$

The black ring is thus related to black holes in four dimensions [16].

In the present context the point is that the extremization principle (132) applies to both electric and magnetic attractors. This provides a thermodynamic interpretation of the attractor mechanism. One obstacle to a complete symmetry between the electric and magnetic cases is that near a magnetic attractor point one must apply (132) with $Q_{I}, q^{I}$, and $R$ fixed, while near an electric attractor it is $Q_{I}$ and $J$ that should be kept fixed. In either case these are the parameters that define the solution.

There is one surprising feature of the proposed physical extremization principle: the entropy is minimized at the attractor point. This may be the correct physics: as one moves closer to the horizon, the geometry is closer to the microscopic data. It is also in harmony with the result that, at least in some cases, extremization over the larger moduli space that includes multi-center configurations gives split attractor flows that correspond to independent regions that have even less entropy [10, with the end of the flow plausibly corresponding to "atoms" that have no entropy at all 23, 24].

We end with a summary of this subsection: we have proposed an extremization principle (132) that applies to both the electric (black hole) and magnetic (black ring) cases. A physical interpretation in terms of thermodynamics looks promising at the present stage of development. In order to fully establish the proposed principle one would need a more detailed understanding of general

\footnotetext{
${ }^{6}$ Although (41) was given in the spherically symmetric case, it can be generalized to include angular momentum [22] (just subtract $J^{2}$ under the square root. The argument given below carries through.

${ }^{7}$ This statement has an obvious alternate version that applies to general CalabiYau spaces.
} 
flows, including those that have magnetic charges, and one would also need a more general definition of charges.

Acknowledgement. I thank Stefano Bellucci for organizing a stimulating meeting and Per Kraus for collaboration on the material presented in these lectures. I also thank Alejandra Castro for reading the manuscript carefully and proposing many improvements, and Josh Davis for discussions.

\section{References}

1. P. Kraus and F. Larsen, "Attractors and black rings," Phys. Rev. D 72, 024010 (2005) [arXiv:hep-th/0503219].

2. S. Ferrara, R. Kallosh and A. Strominger, " $\mathrm{N}=2$ extremal black holes," Phys. Rev. D 52, 5412 (1995) [arXiv:hep-th/9508072]; S. Ferrara and R. Kallosh, "Supersymmetry and Attractors," Phys. Rev. D 54, 1514 (1996) [arXiv:hepth/9602136] "Universality of Supersymmetric Attractors," Phys. Rev. D 54, 1525 (1996) [arXiv:hep-th/9603090]; A. Strominger, "Macroscopic Entropy of $N=2$ Extremal Black Holes," Phys. Lett. B 383, 39 (1996) [arXiv:hepth/9602111].

3. G. W. Moore, "Les Houches lectures on strings and arithmetic," arXiv:hepth/0401049.

4. A. C. Cadavid, A. Ceresole, R. D'Auria and S. Ferrara, "Eleven-dimensional supergravity compactified on Calabi-Yau threefolds," Phys. Lett. B 357, 76 (1995) [arXiv:hep-th/9506144].

5. A. Strominger and C. Vafa, "Microscopic Origin of the Bekenstein-Hawking Entropy," Phys. Lett. B 379, 99 (1996) [arXiv:hep-th/9601029].

6. J. R. David, G. Mandal and S. R. Wadia, Phys. Rept. 369, 549 (2002) [arXiv:hep-th/0203048].

7. A. H. Chamseddine, S. Ferrara, G. W. Gibbons and R. Kallosh, "Enhancement of supersymmetry near 5d black hole horizon," Phys. Rev. D 55, 3647 (1997) [arXiv:hep-th/9610155].

8. A. Strominger, "Black hole entropy from near-horizon microstates," JHEP 9802, 009 (1998) [arXiv:hep-th/9712251].

9. M. Cvetic and F. Larsen, "Statistical entropy of four-dimensional rotating black holes from near-horizon geometry," Phys. Rev. Lett. 82, 484 (1999) [arXiv:hepth/9805146].

10. F. Denef, "On the correspondence between D-branes and stationary supergravity solutions of type II Calabi-Yau compactifications," arXiv:hep-th/0010222; "(Dis)assembling special Lagrangians," arXiv:hep-th/0107152; B. Bates and F. Denef, "Exact solutions for supersymmetric stationary black hole composites," arXiv:hep-th/0304094.

11. G. Lopes Cardoso, B. de Wit and T. Mohaupt, "Corrections to macroscopic supersymmetric black-hole entropy," Phys. Lett. B 451, 309 (1999) [arXiv:hepth/9812082]; "Deviations from the area law for supersymmetric black holes," Fortsch. Phys. 48, 49 (2000) [arXiv:hep-th/9904005].

12. A. Dabholkar, "Exact counting of black hole microstates," Phys. Rev. Lett. 94, 241301 (2005) [arXiv:hep-th/0409148]; A. Dabholkar, F. Denef, G. W. Moore 
and B. Pioline, "Precision counting of small black holes," JHEP 0510, 096 (2005) [arXiv:hep-th/0507014].

13. J. M. Maldacena, A. Strominger and E. Witten, "Black hole entropy in Mtheory," JHEP 9712, 002 (1997) [arXiv:hep-th/9711053].

14. S. Ferrara, G. W. Gibbons and R. Kallosh, "Black holes and critical points in moduli space," Nucl. Phys. B 500, 75 (1997) [arXiv:hep-th/9702103].

15. K. Goldstein, N. Iizuka, R. P. Jena and S. P. Trivedi, "Non-supersymmetric attractors," Phys. Rev. D 72, 124021 (2005) [arXiv:hep-th/0507096].

16. I. Bena and P. Kraus, "Microscopic description of black rings in AdS/CFT," JHEP 0412, 070 (2004) [arXiv:hep-th/0408186]; M. Cyrier, M. Guica, D. Mateos and A. Strominger, "Microscopic entropy of the black ring," Phys. Rev. Lett. 94, 191601 (2005) [arXiv:hep-th/0411187].

17. F. Larsen, "Entropy of thermally excited black rings," JHEP 0510, 100 (2005) [arXiv:hep-th/0505152].

18. J. C. Breckenridge, R. C. Myers, A. W. Peet and C. Vafa, "D-branes and spinning black holes," Phys. Lett. B 391, 93 (1997) [arXiv:hep-th/9602065].

19. H. Elvang, R. Emparan, D. Mateos and H. S. Reall, "A supersymmetric black ring," Phys. Rev. Lett. 93, 211302 (2004) [arXiv:hep-th/0407065]; "Supersymmetric black rings and three-charge supertubes," Phys. Rev. D 71, 024033 (2005) [arXiv:hep-th/0408120].

20. I. Bena and N. P. Warner, "One ring to rule them all ... and in the darkness bind them?," arXiv:hep-th/0408106.

21. K. Copsey and G. T. Horowitz, "The role of dipole charges in black hole thermodynamics," Phys. Rev. D 73, 024015 (2006) [arXiv:hep-th/0505278].

22. R. Kallosh, A. Rajaraman and W. K. Wong, "Supersymmetric rotating black holes and attractors," Phys. Rev. D 55, 3246 (1997) [arXiv:hep-th/9611094].

23. S. D. Mathur, "The fuzzball proposal for black holes: An elementary review," Fortsch. Phys. 53, 793 (2005) [arXiv:hep-th/0502050].

24. P. Berglund, E. G. Gimon and T. S. Levi, "Supergravity microstates for BPS black holes and black rings," JHEP 0606, 007 (2006) [arXiv:hep-th/0505167]; V. Balasubramanian, E. G. Gimon and T. S. Levi, "Four dimensional black hole microstates: From D-branes to spacetime foam," arXiv:hep-th/0606118. 